\title{
A Study to Assess the Physio-Culinary Quality of Chia Seeds
}

\author{
Shweta Singh \\ Assistant Professor, Department of Nutrition and Dietetics, School of Allied healthcare and Sciences, Jain \\ (Deemed to be University), Bengaluru
}

\begin{abstract}
Chia (Salvia hispania l.), constitutes a potential alternative raw material and ingredient in food industry applications. Chia seeds are an abundant source of the n-3 polyunsaturated fatty acid, $\alpha-$ linolenic acid. Alpha-linolenic acid is an essential fatty acid, which, through successive metabolic desaturation and elongation reactions gives rise to dha and epa. EPA and DHA are now widely recognised as having an important role in the prevention of coronary heart disease. Hence, to diversify the use of chia seeds, it was used to develop 4 products, a sweet, a savoury, a drink and a jam in both powdered and raw form. A panel of 25 members was chosen for organoleptic evaluation in order to find the most accepted quantity of chia in each of the products. Nutrition composition of each of these products was enhanced with the addition of chia seeds. Total protein, fat, phosphorous and dietary fibre was increased in all the final variations. The total moisture content of kiwi chia jam was increased due to the addition of chia seeds. Calcium content of chia gel and milk was chemically estimated. The findings showed a $40 \%$ increase in calcium content of chia gel as compared to milk. Kiwi chia jam was compared to standard kiwi jam to test the gelling capability of chia seed as an alternative to citric acid. Statistical average was used to see the percentage increase in terms of acceptability and nutrient enhancement from the basic recipe to the final product.
\end{abstract}

Key Words: chia seeds, potential raw material, essential fatty acid, chia gel

\section{INTRODUCTION}

With increasing public health awareness worldwide, demand for functional food with multiple health benefits has also increased. Seeds from salvia hispanica or more commonly known as chia are a traditional food in Central and Southern America. ${ }^{(1)}$. The seed contains from $25 \%$ to $40 \%$ oil with $60 \%$ of it comprising (omega) $\omega-3$ alphalinolenic acid and $20 \%$ of (omega) $\omega-6$ linoleic acid ${ }^{(4)}$.

Chia seed is composed of protein $(15-25 \%)$, fats $(30-33 \%)$, carbohydrates (26-41\%), high dietary fiber (18-30\%), ash (4-5\%), minerals, vitamins, and dry matter (90-93\%). Another key feature of chia seed is that it does not contain gluten. ${ }^{(1)}$

Chia seeds offer powerful healthbuilding benefits like increased endurance and sustained energy, improving cardio vascular health, mental focus, concentration, stabilizing blood sugar, reducing craving and helping to detoxify the body by super hydration. It is a rich source of antioxidants and fibre which helps to combat diseases like diabetes and cervical cancer. ${ }^{(7)}$

Exposing the chia to liquid gives the seed a sticky, gelatinous consistency, resulting in additional food preparation options. They can be added to smoothies or can be topped on salads. ${ }^{(10)}$

Depending on the likeability and acceptance of chia seeds among the general population, it was decided to use chia seeds in two forms- in the flour form and the raw form. To cover all categories, it was decided to choose 3 forms, a) a sweet item; b) a savoury item; and c) a beverage item to 
check its universal application and its acceptance in the society. Also, to test the gelling capabilities of chia seeds, jams were made. For the development of jams, various fruits were also used ${ }^{(12)}$.

\section{METHOD}

The present study aims at the preparation of chocolate cookies, nachos, mango smoothie and kiwi jam fortified with chia seeds. The product was developed keeping in mind the fact that the consumers should readily accept and afford the products so developed.

Chia-oatmeal cookies and nachos enriched with chia seeds: the chia seeds were slightly roasted and powdered. The basic recipe of the product was made and evaluated using the hedonic scale. Chia powder was added in different quantities and evaluated till it was accepted.

Mango chia smoothie: The chia fortified recipe was prepared and evaluated using the hedonic scale. Chia seeds was added in different quantities and evaluated till it was accepted.

Kiwi chia jam: the basic recipe of the product was made and evaluated using the hedonic scale. Chia powder was added in different quantities and evaluated till it was accepted. Serial dilution was done to check the microbial load. Jam analysis was done for comparison.

\section{RESULTS AND DISCUSSIONS}

It has been proven that chia seeds have a universal application but its usage and consumption seem limited especially in India. To make the benefits of these seeds reach everyone, products in different categories have been developed so that people of all age groups can consume.

Chia-oatmeal cookies: Out of the 6 variations, the last variation was most accepted. The colour, appearance and odour were extremely appreciated. The protein content of the final variation increased by $44 \%$, the fat content increased by $37 \%$ and the energy content increased by $8 \%$ as compared to the basic recipe. It is seen that the calcium, phosphorous as well as the dietary fibre content of the cookies have risen sharply when chia seeds were incorporated in them. There is a 5 times difference between calcium content of the basic recipe and the final variation and more than 4times difference between the phosphorous content of the basic recpe and the final product. Again, the basic recipe had no fibre present in it, with the use of oats and chia seeds, the product now have fibre present in it.

Nachos enriched with chia seeds: Variation 2 was seen to be the most accepted. It scored the maximum in all the departments. The colour, appearance and odor were extremely appreciated. The taste was better than the other variations. The quantity of chia seeds included in this variation was accepted and it did not change the colour of the nachos significantly.

The protein content increases by $23 \%$, the fat increased almost by $92 \%$, the carbohydrate increased by $9 \%$ and the energy increased by $23 \%$ in variation 2 as compared to the basic recipe. This percentage increase from the basic recipe is mainly because of the use of chia seeds. Since, chia seeds contain omega-3 fatty acids, the fat content increased. . It was seen that there is a $74 \%$ increase in the fibre content. This is again because of the addition of the chia seeds. The calcium content as well as the phosphorous content significantly increases in variation 2 as compared to the basic recipe. The calcium content is almost 16 times and the phosphorous content is almost 20 times as compared to the basic recipe.

Mango chia smoothie: Variation 3 was seen to be the most accepted. It scored the maximum in all the categories. The colour, appearance and odour were extremely appreciated. The taste was better than the other variations. The quantity of chia seeds included in this variation was accepted and it did not stick in the mouth of the panel members. It was giving the smoothie the desired crunchy mouth feel. The protein increased by $21 \%$, carbohydrate increased 
by $4 \%$ and the energy increased by 10 percent in variation 4 as compared to the basic recipe. There was a 7 fold increase in the fat content. Chia seeds are known for its omega-3 fatty acid content. Hence the fat content rose significantly by variation 3 and decreased a little by variation 4 . The calcium content increased by $25 \%$ whereas the phosphorous content significantly gets increased by $50 \%$ in variation 4 as compared to the basic recipe. The fibre content increases 4 times when chia seeds are added to the smoothie.

Kiwi chia jam: The sensory evaluation of jam was done in 2 ways. One without bread and the other one with bread. Variation 3 was seen to be the most accepted in both the evaluations. It scored the maximum in all the categories. The colour, appearance and odour were extremely appreciated. The taste was better than the other variations. The quantity of chia seeds included in this variation was accepted and it did not stick in the mouth of the panel members. Kiwifruit does not store well because of their high moisture content and soft texture.

It is seen that that kiwi chia jam contains a high amount of insoluble solids as compared to the standard kiwi jam. This leads to a higher amount of total solids. The levels of reducing are indicative of the quality of these food products. The strength of the kiwi jam before inversion was $262.82 \mathrm{mg} / \mathrm{ml}$ whereas for the jam with chia seeds were $72.47 \mathrm{mg} / \mathrm{ml}$. The strength of the kiwi jam after inversion was $3.7 \mathrm{mg} / \mathrm{dl}$ whereas for the jam with chia seeds were $24.076 \mathrm{mg} / \mathrm{ml}$.

The moisture percentage of the kiwi chia jam is slightly higher than that of the standard kiwi jam. There is difference of mere $7 \%$.

Calcium content of chia gel and milk: Recent studies have revealed that chia (salvia hispanica) is a remarkably wellrounded nutritional source, providing protein, magnesium, phosphorus, and essential fatty acids. Gluten-free and grainfree, chia also has six times the calcium of milk. Hence to prove this finding, the calcium content per $100 \mathrm{gms}$ was calculated for milk, chia gel and each of the products like cookies, nachos, smoothie and jam. The calcium content was taken out by titrimetric method. There is a $40 \%$ difference in the calcium content of chia gel and milk. Since, the mango smoothie contains both milk and chia, it has the highest amount of calcium as compared to all the 4 product made. The calcium content of the smoothie is almost 3 times as compared to the calcium content in nachos, mainly because of the quantity of the chia seeds and the ingredients used.

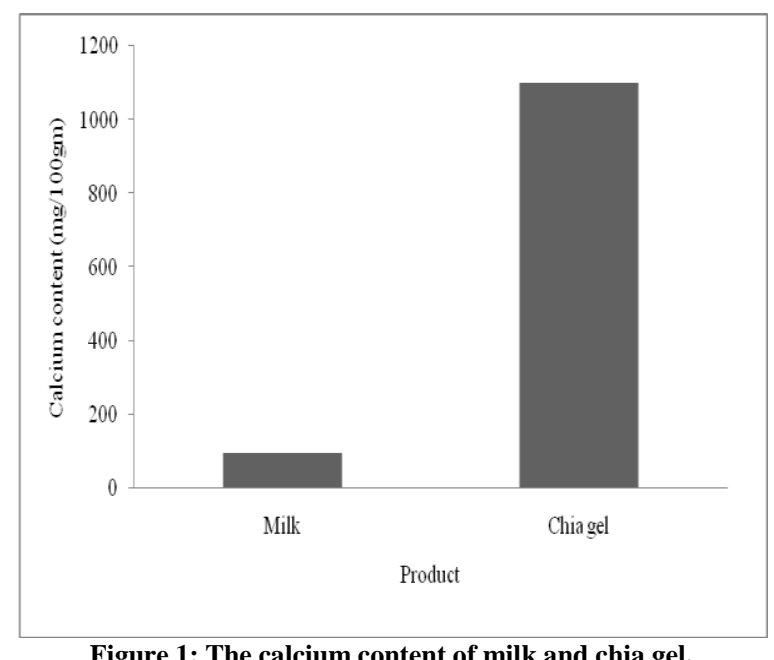

Figure 1: The calcium content of milk and chia gel.

\section{SUMMARY AND CONCLUSION}

Chia seeds are an abundant source of the n-3 polyunsaturated fatty acid, $\alpha$ linolenic acid. Alpha-linolenic acid is an essential fatty acid, which, through successive metabolic desaturation and elongation reactions gives rise to dha and epa. Epa and dha are now widely recognised as having an important role in the prevention of coronary heart disease. Hence, to diversify the use of chia seeds, it was used to develop 4 products, a sweet, a savoury, a drink and jam

Chia-oatmeal cookies: On comparing the variations against the basic recipe in the field of appearance, color, taste, texture, odour and overall rating, it was seen that variation 6 was the most accepted by all the panel members. This variation contained $35 \mathrm{gms}$ of chia seeds, $120 \mathrm{gms}$ of oats and 30 gms of maida. The chia seeds perfectly blended with the cookies, without giving an 
aftertaste of sticking to the teeth. With the help of chocolate chips the appearance was also accepted. The cost per biscuit was rs. 2.049 .

Nachoes enriched with chia seeds: Apart from the basic recipe, 2 variations were prepared with different amounts of chia and spices. On comparing the variations against the basic recipe in the field of appearance, color, taste, texture, odour and overall rating, it was seen that variation 2 was the most accepted by all the panel members. The complain of chia getting stuck or the taste being too bland was removed in this variation. Hence, variation 2 was chosen as the best variation.

Mango chia smoothie: 4 variations were prepared by variating the chia seeds. Since it is a beverage, whole chia seeds were used. Chia seeds, when in contact with a liquid swell up and forms a gel like structure. Variation 3 was disliked by majority of the panel members as the amount of chia used was the maximum which was disliked by the panel members. Variation 4, had a perfect yellow colour with just the desirable and the acceptable amount of chia seeds. Hence, the best variation was variation 4 and on comparing the variations against the basic recipe in the field of appearance, color, taste, texture, odour and overall rating, variation 4 scored the most.

Kiwi chia jam: On comparing the variations against the basic recipe in the field of appearance, color, taste, texture, odour and overall rating, it was seen that variation 3 and variation 6 was the most accepted by all the panel members. The variation 3 contained $7.5 \mathrm{gms}$ of chia seeds, $100 \mathrm{gms}$ of kiwi and 60 gms of sugar while variation 6 contained and extra of citric acid. The chia seeds perfectly blended with the jam and also helped the jam to set in variation3, without giving an aftertaste or sticking to the teeth. Since, chia seeds successfully helped the jam to set, variation 3 was considered to be the most acceptable variety of all. The acidity, inverted sugar content and soluble solids was less as compared to the standard jam. On the other hand, the percentage of moisture was higher

\section{Acknowledgement: None}

\section{Conflict of Interest: None}

\section{Source of Funding: None}

\section{REFERENCES}

1. Ali N, Amerman D "The Promising Future of Chia, Salvia hispanica L.", Hindawi Publishing Corporation Journal of Biomedicine and Biotechnology, Volume 2012, Article ID 171956, 9 pages

2. Aguilera J., Garcia A., Rubio O., "Mucilage from chia seeds (salvia hispanica) : microstructure, Physico-chemical characterization and applications in food industry", Santiago de Chile, July, 2012. 12

3. Ancona D., Campos M., Guerrero L., Rubio G., Solís N., "Physicochemical characterization of chia (Salvia hispanica) seed oil from Yucatán, México", Vol.5, No.3, 220-226 (2014)

4. Anschuetz, N. F. 1996. "Evaluating Ideas and Concepts for New Consumer Products," in The PDMA Handbook of New Product Development, Rosenau, M. D., A. Griffin, G. A. Castellion, and N. F. Anschuetz, eds. New York: John Wiley \& Sons, pp. 195206.

5. Ayerza, R., Coate W "Commercial production of Chia in Northwestern Argentina", Journal of the American Oil Chemists' Society, 75:1417-1420.

6. Ayerza R., Coates W., " Effect of dietary $\alpha$ linolenic fatty acid derived from chia when fed as ground seed, whole seed and oil on lipid content and fatty acid composition of rat plasma. Journal of Nutrition and Metabolism.", 2007; Vol 51(1).

7. Bird E.W, Webber J., Determination of Calcium and Magnesium in Milk by E.D.T.A. Titration”, Department of Dairy and Food Industry, Iowa State University, Ames, Iowa, Volume 44, Issue 6, Page 10361049

8. Bresson J., Flynn A., etal., "Opinion on the safety of "Chia seeds (Salvia hispanica L.) and ground whole Chia seeds' as a food ingredient", The European Food Safety Authority Journal (2009) 996, 1-26. 
9. Coelho M., Salas-Mellado M., "Chemical Characterization of CHIA (Salvia hispanica L.) for Use in Food Products", Journal of Food and Nutrition Research, 2014, Vol. 2, No. 5, 263-269

10. Craig R., "Application for Approval of Whole Chia (Salvia hispanica L) Seed and Ground Whole Chia as Novel Food Ingredients", 76 Old Portglenone Road, Ahoghill,Co. Antrim, Northern Ireland, BT42 1LQ

11. Gopalan C.,Sastri Rama.,'Ntritive Value Of Indian Foods", National Institute of
Nutrition, Indian Council of Medical Research, 1989.

12. Kumar U., Rangaraju A., 'A Pharmacognostic Study on Salvia Hispanica', American Journal of Pharmacy and Health Research, 2013, Volume 1, Issue 9,ISSN : 2321-3647.

How to cite this article: Singh S. A study to assess the physio-culinary quality of chia seeds. Int J Health Sci Res. 2021; 11(7):119-123. DOI: https://doi.org/10.52403/ijhsr.20210717 